\section{DC defects in HIV-1}

Patients infected with human immunodeficiency virus type 1 (HIV-1) often have dysfunctional dendritic cells (DCs), but as HIV-1 cannot directly infect these cells, the basis of this effect has been unclear. In the Journal of Clinical Investigation, Bhardwaj and colleagues show that apoptotic microparticles generated from lymphocytes mainly during acute HIV infection are responsible for this DC dysfunction. Serum, but not virus, isolated from $\mathrm{HIV}^{+}$patients suppresses the Toll-like receptor (TLR)-mediated release of inflammatory cytokines by DCs. This effect on DCs seems to be HIV specific, because serum from patients acutely infected with hepatitis virus $C$ or $B$ is not inhibitory. Furthermore, microparticles in serum from uninfected patients are also unable to modulate DC function, which suggests that microparticles associated with HIV infection are somehow qualitatively distinct. Although the identity of the microparticle protein(s) remains unclear, CD44 expression by DCs is critical for its recognition and for mediation of the modulatory signal.

J. Clin. Invest. 122, 4685-4697 (2012)

\section{Nonprofessional phagocytes}

The engulfment of apoptotic cells by professional phagocytes can elicit anti-inflammatory mediators and dampen inflammation in the tissues. In Nature, Ravichandran and colleagues show that airway epithelial cells are able to engulf apoptotic targets and produce anti-inflammatory mediators. Deletion of the small GTPase Rac1 in airway epithelial cells results in defective clearance of apoptotic cells and less production of the anti-inflammatory cytokines IL-10 and TGF- $\beta$. In addition, airway epithelial cells are more sensitive to allergic airway inflammation. Rac1deficient epithelial cells produce more IL-33 after allergen challenge, which increases the number of nuocyte-like cells in the lungs. These data indicate that airway epithelial cells are major players in modulating tolerance to common airway allergens.

IV

Nature (12 December 2012) doi:10.1038/nature11714

\section{Cereals activate TLR4}

Gluten in wheat, barley and rye can induce pathology in the small intestine, such as celiac disease, due to the immunogenicity of several nondegraded gluten peptides that trigger activation of T cells in susceptible people who express HLA-DQ2 and HLA-DQ8. In the Journal of Experimental Medicine, Junker et al. show that $\alpha$-amylase-trypsin inhibitors (ATIs), which have a role in pest resistance in cereals, are strong inducers of innate immune responses in mice and humans. ATIs engage the TLR4MD2-CD14 complex and elicit inflammatory cytokines such as interleukin 8 (IL-8), IL-12, TNF, CCL2 and CCL5 in DCs, monocytes and macrophages and induce intestinal inflammation in mice deficient in recombination-activating gene 1 . ATls induce a similar degree of activation in DCs from healthy controls and patients with celiac disease, which indicates that this activating interaction might be controlled in healthy people.

J. Exp. Med. 209, 2395-2408 (2012)

\section{Maturing pDCs}

Plasmacytoid DCs (pDCs) have important antiviral functions due to their ability to produce vast amounts of type I interferon. In Blood, Kaisho and colleagues identify an important cell-intrinsic role for Spi-B (a member of the Ets family of transcription factors) in pDC function. Spi-B has high expression in pDCs, but so far its precise role has been unclear. In addition, the transcription factor E2-2 is known to be critical for early pDC development. The authors find that Spi-B associates and acts in synergy with the type I interferon-inducing 'master' transcription factor IRF7. Spi-B-deficient mice show a complex pattern of development, with fewer mature bone marrow pDCs, but this effect is less apparent for peripheral pDCs. However, Spi-B-deficient pDCs have impaired type I interferon responses to stimulation of TLR7 and TLR9. Transcriptional profiling suggests that Spi-B regulates a set of genes that overlaps but is distinct from those regulated by E2-2, with Spi-B being involved chiefly in the functional maturation of pDCs.

Blood 120, 4733-4743 (2012)

\section{Macrophage Jnk}

The infiltration of adipose tissues and elaboration of proinflammatory cytokines by macrophages is associated with the development of metabolic disease. In Science, Han et al. show selective loss of Jnk kinase activity in macrophages is sufficient to maintain sensitivity to insulin and glucose in mice fed high-fat diets. Fewer Jnk-deficient macrophages than wildtype macrophages accumulate in adipose tissues. Those Jnkdeficient macrophages have lower expression of M1-associated proinflammatory cytokines and chemokines; however, loss of Jnk activity does not impair the M2 polarization of macrophages. These findings suggest that Jnk signaling in macrophages is responsible for the $\mathrm{M} 1$ polarization of macrophages and their recruitment to adipose tissues. Inhibition of Jnk activity in macrophages may therefore suppress the inflammation that occurs in diet-induced metabolic syndrome.

\title{
NLRP3 ubiquitination
}

Inflammasomes are activated in response to various microbial or damage-associated signals, which leads to the release of pyrogenic IL-1 $\beta$. In Molecular Cell, Yuan and colleagues show that the inflammasome component NLRP3 is regulated by ubiquitin modification. Pharmacological inhibitors of deubiquitinase block NLRP3-dependent activation of caspase- 1 and secretion of IL- $1 \beta$ in activated macrophages, but NLRC4- or AIM2-dependent inflammasomes are unaffected by such treatment. Ubiquitin Lys 48 and Lys63 chains decorate the NACHT and leucine-rich repeat domains of NLRP3, but the stability of NLRP3 protein does not seem to be affected. These ubiquitin modifications must be removed before assembly of the inflammasome with ASC and pro-caspase-1. The deubiquitinase BRCC3 can activate NLRP3 by removing ubiquitin chains in vitro. Knockdown of BRCC3 by small interfering RNA in activated macrophages prevents NLRP3 inflammasome-mediated release of IL- $1 \beta$. These findings point to another level of regulation of NLRP3 inflammasome activity that potentially can be targeted therapeutically.

$L A D$

Written by Laurie A. Dempsey, Zoltan Fehervari \& Ioana Visan 